\title{
Oriented inorganic perovskite absorbers processed by colloidal-phase fumigation
}

\author{
Ziren Zhou ${ }^{1 \dagger}$, Hong Wei Qiao ${ }^{1 \dagger}$, Xiaolong $\mathrm{Li}^{2}$, Chen $\operatorname{Lin}^{3}$, Jin Xie ${ }^{1}$, Yiheng Shi ${ }^{1}$, Zeqing Lin ${ }^{1}$, \\ Bing $\mathrm{Ge}^{1}$, Mengjiong Chen ${ }^{1}$, Yi Zheng Jin ${ }^{3}$, Shuang Yang ${ }^{1 *}, \mathrm{Yu} \mathrm{Hou}^{1 *}$ and Hua Gui Yang ${ }^{1}$
}

\begin{abstract}
Inorganic perovskite absorbers are promising candidates for next-generation photovoltaics due to their good thermal and light stabilities. Halide-mixed $\mathrm{CsPbI}_{x} \mathrm{Br}_{3-x}$ reach a compromise between its structural tolerance and absorption edge, yet the power conversion efficiencies (PCEs) of the asfabricated cells can be considerably limited by the nonideal quality of solution-processed films. Here we demonstrated a fumigation strategy on colloidal perovskite films using dual$O$-donor ethyl acetate (EA). By in-situ monitoring this stage with grazing-incidence wide-angle $\mathrm{X}$-ray scattering technology, we reveal that EA fumigation would impose ripening barrier on colloidal inorganic perovskites and hence slow down the nucleation rate, leading to an intermediate state for processing high-crystallinity and oriented perovskite films with improved photophysical properties. An optimized PCE of $16.6 \%$ was finally yielded upon wide-bandgap $(1.9 \mathrm{eV})$ perovskite absorber.
\end{abstract}

Keywords: cesium lead halide perovskite, fumigation, orientation, photophysical properties, solar cells

\section{INTRODUCTION}

Inorganic perovskite solar cells (PSCs) have distinct advantages toward robust light harvesting, of which the record power conversion efficiencies (PCEs) have been continuously broken in recent years [1-4]. The quality of solution-processed $\mathrm{CsPbI}_{x} \mathrm{Br}_{3-x}$ films, which can determine the ultimate performance of devices, is strongly dependent on the nucleation and growth stages. In this regard, a tailored crystallization condition is in particular demand for obtaining the high-quality perovskite films with desired photophysical properties. To date, a plethora of methods targeting crystallization optimization have been demonstrated efficacious to enhance the quality of inorganic perovskite films, such as gradient thermal annealing $[2,5]$, hot air purge $[3,6,7]$, substrate optimization [8], and elemental doping [3,9-13], which give rise to good photovoltaic performance of PSCs.

A high-quality perovskite film should typically feature high crystallinity, large grain size and reduced macroscopic defects (e.g., pinhole). As a complementarity to those features, the mesoscopic arrangement of perovskite lattices (also refer to the orientation) is an important factor that can basically determine the electrical properties of polycrystalline films [14]. Several chemical strategies have been employed to create the oriented structure upon organic-inorganic hybrid perovskite films, such as the introduction of methylammonium chloride ( $\mathrm{MACl}$ ) [15], lead chloride $\left(\mathrm{PbCl}_{2}\right)$ [14], and ammonium thiocyanate $\left(\mathrm{NH}_{4} \mathrm{SCN}\right)$ to precursors [16]. In addition, using pre-heated substrate, Tsai et al. [17] reported a hotcasting method to fabricate low-dimensional perovskite films with ordered distribution of quantum wells. The textured perovskite films in those cases were clearly identified by the grazing-incidence wide-angle X-ray scattering (GIWAXS) patterns along the out-of-plane direction. Such lattice alignment renders anisotropic carrier mobility as well as reduced trap-state density to perovskite films [14], which is of significance to their photovoltaic applications.

Here we fabricated the wide-bandgap inorganic perovskite absorbers by fumigating the colloidal films with ethyl acetate (EA) vapor. Despite the solvent-vaporassisted crystallization has widely been employed to

\footnotetext{
${ }^{1}$ Key Laboratory for Ultrafine Materials of Ministry of Education, Shanghai Engineering Research Center of Hierarchical Nanomaterials, School of Materials Science and Engineering, East China University of Science and Technology, Shanghai 200237, China

${ }^{2}$ Shanghai Synchrotron Radiation Facility, Shanghai Institute of Applied Physics, Chinese Academy of Sciences, Shanghai 201204, China

${ }^{3}$ Department of Chemistry, Zhejiang University, Hangzhou 310058, China

$\uparrow$ These two authors contributed equally to this work.

* Corresponding authors (emails: yhou@ecust.edu.cn (Hou Y); syang@ecust.edu.cn (Yang S))
} 
optimize the film quality of polymers and hybrid perovskites [18-21], it has yet to be reported for inorganic perovskites, especially targeting the orientation regulation. With the assistance of in-situ GIWAXS facility, we found that the EA atmosphere would retard the nucleation of perovskite species at low-temperature annealing stage, in contrast to the faster nucleation rate in nitrogen. The as-fumigated colloidal state facilitates the formation of highly crystallized perovskite films with an oriented lattice structure. An improved electrical conductivity was achieved on fumigated perovskite film, accompanied by the mitigated surface potential losses under light illumination. PSCs based on such textured perovskite films yielded a stabilized PCE of $16.3 \%$ under simulated AM $1.5 \mathrm{G}$ irradiation.

\section{EXPERIMENTAL SECTION}

\section{Materials}

Dimethyl sulfoxide (DMSO, anhydrous 99.9\%), cesium iodide (CsI, 99.9\%), bis(trifluoromethylsulfonyl)imide lithium salt (Li-TFSI, 99.95\%), 4-tert-butylpyridine ( $t$-BP, 99\%) and acetonitrile (anhydrous, 99.8\%) were purchased from Sigma-Aldrich. Lead iodide $\left(\mathrm{PbI}_{2}, 99.9985 \%\right)$ was purchased from Alfa Aesar. Lead bromide $\left(\mathrm{PbBr}_{2}, 98+\%\right)$ was purchased from STREM. EA (GC, 99.9\%) and chlorobenzene (anhydrous, 99.5\%) were purchased from Aladdin. Poly(3-hexylthiophene-2,5-diyl) (P3HT) was purchased from Xi'an Polymer Light Technology. All these materials were used as received.

\section{Device fabrication}

Electron transport layers (ETLs) were prepared by immersing the cleaned fluorine-doped tin oxide (FTO) substrates in aqueous $\mathrm{TiCl}_{4}\left(0.04 \mathrm{~mol} \mathrm{~L}^{-1}\right)$ at $70^{\circ} \mathrm{C}$ for $60 \mathrm{~min}$. The substrates were then washed with deionized water and ethanol, followed by annealing at $500^{\circ} \mathrm{C}$ for $60 \mathrm{~min}$ to form the compact $\mathrm{TiO}_{2}$ layer. The $\mathrm{CsPbI}_{1.8} \mathrm{Br}_{1.2}$ precursors $\left(1.2 \mathrm{~mol} \mathrm{~L}^{-1}\right.$ in DMSO) were spin-coated on ETL at $1000 \mathrm{rpm}$ for $5 \mathrm{~s}$ and $3000 \mathrm{rpm}$ for $30 \mathrm{~s}$, and the colloidal films were baked on a hot plate at $43^{\circ} \mathrm{C}$ in nitrogen or EA atmosphere for $200 \mathrm{~s}$. The EA atmosphere was created by putting the solvent source in a semicovered vessel under the same annealing condition. After fumigation, the films were quickly transferred to another hot plate and annealed at $180^{\circ} \mathrm{C}$ for $10 \mathrm{~min}$. P3HT solutions $(15 \mathrm{mg}$ in $1 \mathrm{~mL}$ chlorobenzene, with $45 \mu \mathrm{L}$ Li-TFSI solution $(2 \mathrm{mg}$ in $1 \mathrm{~mL}$ acetonitrile) and $10.2 \mu \mathrm{L} t$-BP) were then spin-coated on perovskite films at $3000 \mathrm{rpm}$ for $30 \mathrm{~s}$ as the hole transport layer (HTL), followed by an- nealing at $120^{\circ} \mathrm{C}$ for $10 \mathrm{~min}$. Finally, $100-\mathrm{nm}$-thick $\mathrm{Au}$ was thermally evaporated onto the HTL as metal electrodes. All steps were carried out in the nitrogen glove box $\left(\mathrm{H}_{2} \mathrm{O},<0.01 \mathrm{ppm} ; \mathrm{O}_{2},<0.01 \mathrm{ppm}\right)$.

\section{Characterization}

The mass losses of perovskite precursors and EA solvent were measured by a Thermogravimetric Analyzer (NETZSCH, TG209F1-GC) in nitrogen atmosphere. Nuclear magnetic resonance (NMR, Bruker Avance III $400,400 \mathrm{MHz}$ ) equipped with super conducting Fourier spectrometer was used to acquire the ${ }^{1} \mathrm{H}-\mathrm{NMR}$ spectra of perovskite powders at room temperature. The morphology of perovskite films was characterized by field emission scanning electron microscopy (FESEM, HITACHI S4800). X-ray diffraction (XRD) patterns were acquired by Powder XRD (Bruker Advance D8 X-ray diffractometer $\mathrm{Cu}$ Ka radiation, $40 \mathrm{kV}$ ). A Cary $500 \mathrm{UV}$ Vis-NIR spectrophotometer was applied to measure the optical absorption spectra of the perovskite films. Steadystate and time-resolved photoluminescence (PL) spectra were characterized by a Fluorolog-3-p spectrophotometer. The ex-situ and in-situ GIWAXS data were collected at B1-beamline of Shanghai Synchrotron Radiation Facility (SSRF). The monochromatic X-rays (energy $=12.7 \mathrm{eV}$, wavelength $=0.124 \mathrm{~nm}$ ) with $0.2^{\circ}$ and $1^{\circ}$ incident angles were adopted for in-situ and ex-situ 2D scattering, respectively. The sample-to-detector distance of GIWAXS was set to be $294 \mathrm{~mm}$ and the patterns were recorded by a Mar345 CCD camera (marXperts). For insitu GIWAXS characterization, the patterns were scanned and recorded with a time interval of $10 \mathrm{~s}$ in a nitrogenfilled apparatus. The nitrogen atmosphere was created by injecting high-purity nitrogen (99.999\%) into the kaptonsealed apparatus with a constant velocity and the outlet was designed to keep the atmospheric pressure inside the apparatus. The atomic force microscopy (AFM) and Kelvin probe force microscopy (KPFM) images of perovskite films were simultaneously obtained by using $\mathrm{Cy}$ pher atomic force microscopy (Asylum research) in nitrogen atmosphere. The double modulation scheme with tip (radius at the apex below $30 \mathrm{~nm}$ ) was adopted for signal collection: the mechanically fundamental resonance $(\sim 75 \mathrm{kHz})$ for topographic imaging and second resonance $(\sim 75 \mathrm{kHz})$ for alternating current (AC) modulation. The illumination was provided by the equipped green laser source $(520 \mathrm{~nm})$. The solar cells were illuminated by a solar light simulator (Solar IV-150A, Zolix) and light intensity was calibrated by a standard Newport calibrated KG5-filtered Si reference cell. The photo- 
current density-voltage $(J-V)$ curves of devices were measured by Keithley 2400 digital sourcemeter under AM $1.5 \mathrm{G}$ irradiation $\left(100 \mathrm{~mW} \mathrm{~cm}^{-2}\right)$. Devices were masked with a metal aperture to define the active area of $0.0625 \mathrm{~cm}^{2}$. External quantum efficiency (EQE) spectra of solar cells were measured by a Newport-74125 system. Impedance spectra were acquired by the electrochemistry workstation (Parstat 2273, Princeton). The transient photocurrent/photovoltage decays (TPC/TPV) of solar cells were measured by digital storage oscilloscope (KEYSIGHT, DSOX3104T) under the excitation of nitrogen laser (337 nm, SRS NL100).

\section{RESULTS AND DISCUSSION}

Thermal gravimetric analysis (TGA) curves show that EA solvent is a strongly volatile component under the annealing temperature of $43^{\circ} \mathrm{C}$, while the perovskite precursors (in DMSO) are involatile (Fig. S1). According to the miscible nature of EA and DMSO, we infer that EA molecules can be absorbed into the colloidal perovskite film during the low-temperature annealing stage, and may therefore affect the crystallization of perovskites during the following high-temperature annealing. Notably, no signal of EA molecule is observed from the ${ }^{1} \mathrm{H}$ NMR spectra of the as-collected perovskite powders (Fig. S2), suggesting that EA molecules can be totally removed from the ultimate perovskite films.

The morphologies of the as-prepared perovskite films without and with fumigation (designated as reference and fumigated, respectively) were studied by SEM. The fumigated film shows buried grain boundaries (GBs) within a large scanning range, in contrast to the apparent GBs of the reference one (Fig. 1a, b and Fig. S3). A largely enhanced crystallinity of about three times was achieved upon the fumigated perovskite film with respect to the reference one, which was determined by the intensity of $(020) /(101)$ facets from the XRD patterns (Fig. 1c). Note that the room-temperature phase of $\mathrm{CsPbI}_{x} \mathrm{Br}_{3-x}$ should belong to orthorhombic [22].

To unveil the orientational characteristics of perovskite films, we performed GIWAXS along the out-of-plane direction. The distinct (002) and (004) spots in GIWAXS patterns confirm that the crystal domains of both the reference and fumigated perovskite films are mainly oriented along the [001] direction (Fig. 1d, e and Fig. S4), i.e., perpendicular to the substrates. More sharp and discrete Bragg spots, such as $(h k 2)$ and ( $h k 4)$ facets, emerge in the pattern of the fumigated film. Such singlecrystal-like feature indicates an oriented perovskite structure throughout the polycrystalline film [14], probably originating from the parallel-aligned lattices. Besides, we found that when EA is altered by methanol (MT),
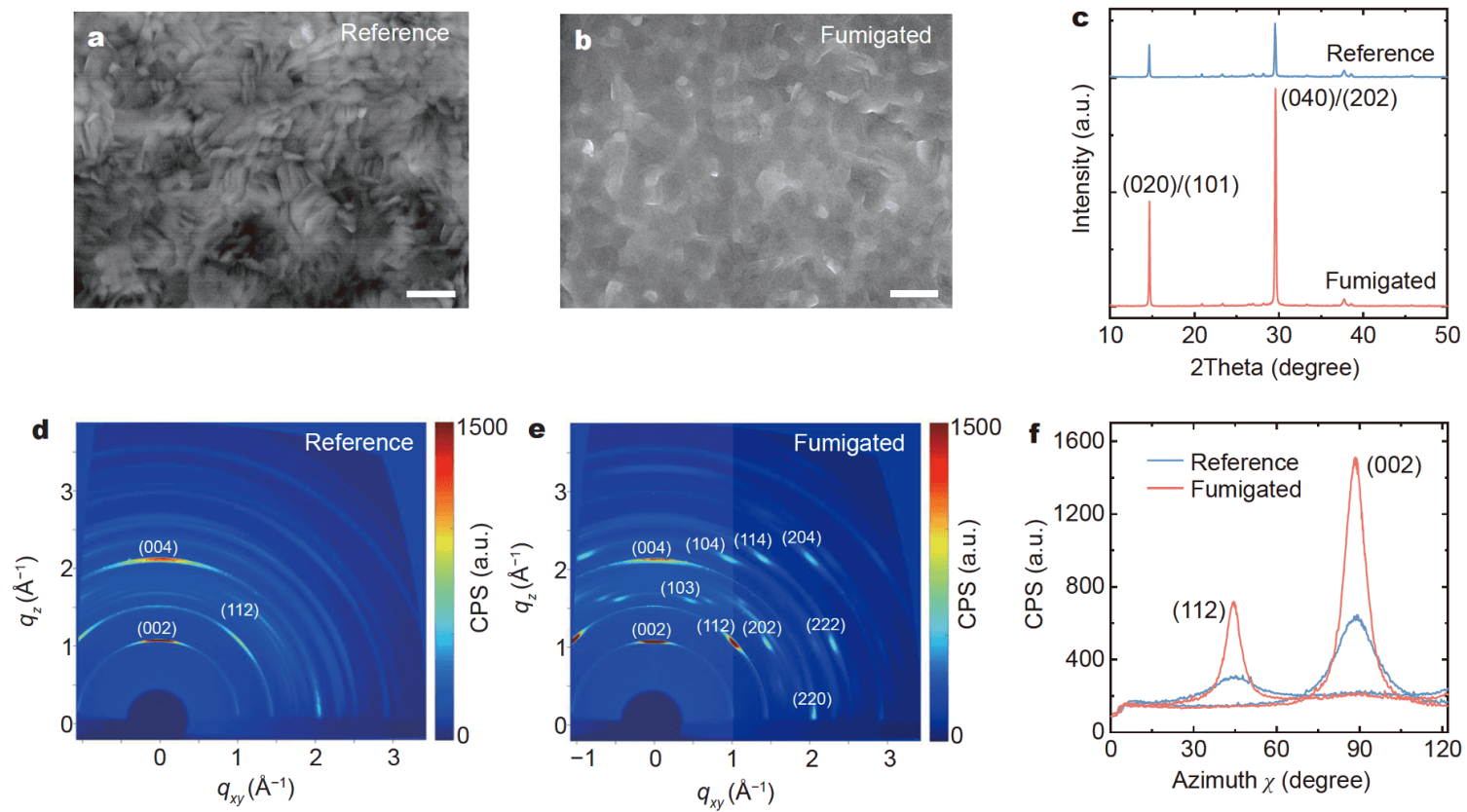

Figure 1 Top-view SEM images of the (a) reference and (b) fumigated perovskite films. All scale bars: $3 \mu \mathrm{m}$. (c) XRD patterns of the reference and fumigated perovskite films. Ex-situ GIWAXS patterns of the (d) reference and (e) fumigated perovskite films along the out-of-plane direction. (f) Azimuthally integrated plots along the rings where (002) and (112) locate. All films were deposited on glass/FTO/TiO ${ }_{2}$ substrates. 
ethanol (ET), isopropanol (IPA) or toluene (TL), the fumigated perovskite films show optimized morphology as well as improved crystallinity, yet with negligible oriented feature to be observed (Figs S5-S7). The fundamental properties of the as-used solvents have been provided in Table S1. We further quantitatively analyzed the integrated intensities of (002) and (112) facets from GIWAXS patterns at azimuthal cuts (125 degree). The counts per second (CPSs) of (002) and (112) facets of the fumigated film are about 2.34 and 2.30 times higher than that of the reference one (Fig. 1f), accompanied by the reduced full-width at half-maximum (FWHM) of peaks (Fig. S8), again verifying its high crystal quality as well as the increased grain size which has been observed from SEM images. The signal influence of the substrate (glass/ $\mathrm{FTO} / \mathrm{TiO}_{2}$ ) has been pre-subtracted from all GIWAXS patterns.

Using in-situ GIWAXS technology, we try to reveal the impact of fumigation on the nucleation of colloidal perovskite species. The CPS evolutions of films in the home- made facility are monitored with a settled time interval of $10 \mathrm{~s}$ within $200 \mathrm{~s}$ (Fig. 2a-c and Fig. S9), equal to the lowtemperature annealing time for device fabrication. The azimuthally integrated CPS of (002) facets show that the ripening of the fumigated colloidal film saturates later than the reference one (in pure nitrogen), of which the normalized data are presented in Fig. 2d. Besides, we found that the fumigated film exhibits a much lower integrated CPS over the entire monitoring time and finally leads to an intermediate state with lower crystallinity (Fig. S10), indicating the retarding effect of fumigation on perovskite ripening. Note that the CPS gap at the starting point $(0 \mathrm{~s})$ should probably due to the set-up time for synchrotron radiation source to produce beamline, during which the retarding function of EA molecules may have already commenced.

To quantitively evaluate the early-stage nucleation rate of colloidal perovskite films, we use the Sharp-Hancock presentation which is described by the relation between the CPS peak intensity $(\alpha)$ and monitoring time $(t)[23]$ :
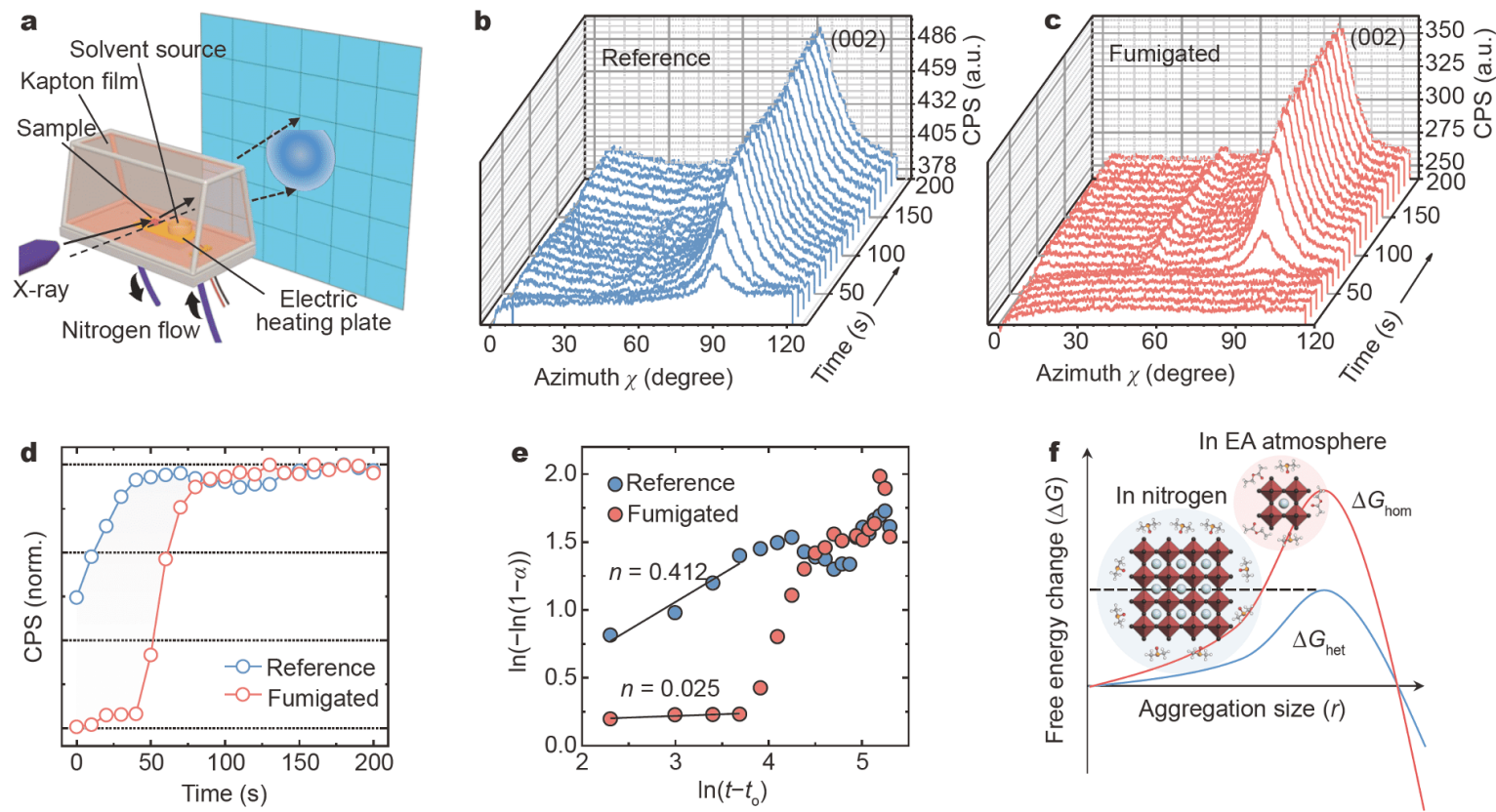

g
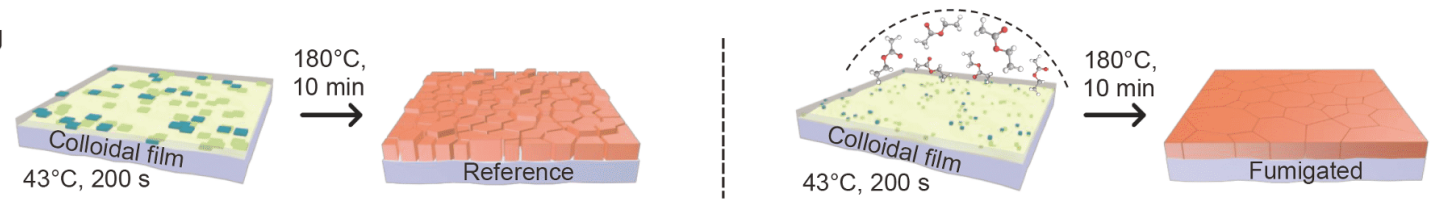

Figure 2 (a) Schematic diagram of in-situ GIWAXS facility. Azimuthally integrated in-situ GIWAXS patterns of the (b) reference and (c) fumigated colloidal perovskite films. All films were deposited on amorphous glass substrates. (d) CPS evolution of (002) facets relative to the monitoring time. (e) CPS evolution of (002) facets plotted versus the monitoring time in the Sharp-Hancock presentation. (f) Conceptual nucleation model of colloidal perovskites in nitrogen and EA atmospheres. The free energy changes $(\Delta G)$ for homogeneous and heterogeneous nucleations are marked with $\Delta G_{\text {hom }}$ and $\Delta G_{\text {het }}$, respectively. (g) Schematic diagrams of liquid-to-solid transformation of colloidal perovskite films under different atmospheres. 
$\ln \{-\ln [1-\alpha(t)]\}=n \ln \left(t-t_{0}\right)+n \ln (k)$,

where $n$ and $k$ are the slope and intercept of linearly fitted data, respectively. As shown in Fig. 2e, the $n$-value of the fumigated film $(0.025)$ is significantly smaller than the $n$ value of the reference one (0.412), suggesting a large nucleation barrier enabled by fumigation [23]. Combining classical nucleation theory, we are able to conjecture a ripening model for colloidal perovskites under routine and fumigated conditions. The as-adsorbed EA molecules might energetically impose higher barrier on perovskite nucleation, so as to slow down its rate and trigger the homogeneous nucleation (Fig. 2f). This also agrees with the morphological results of the fumigated perovskite film, i.e., owning larger grain size than the heterogeneously nucleated reference one. One possible reason for such behavior may be due to the adsorption of EA molecules onto the nuclei surface through the interaction of dual-O-donors with metal cations (e.g., $\mathrm{Pb}^{2+}$ ) (Fig. S11), yet the in-depth reasons remain to be explored. The liquid-to-solid transformations of colloidal perovskite films in nitrogen and EA atmospheres are schematically presented in Fig. 2g.

The photophysical properties of perovskite films were studied by KPFM under both dark and illumination conditions (Fig. S12). The contact potential difference (CPD), which equals to the work function $\left(W_{\mathrm{F}}\right)$ of film surface relative to the tip, can be used to assess the surface potential losses of perovskite films [24,25]. As shown in
Fig. 3a-d, the CPD maps of the films are geometrically consistent with their height profiles (Fig. S13). The statistical results of CPD maps show that the fumigated film has higher CPD than the reference one under both dark and illuminating conditions (Fig. 3e), which corresponds to its lower $W_{\mathrm{F}}$. This means that more charged carriers are accumulated on the surface of the fumigated perovskite film [26]. Besides, according to the most recent work [27], the CPD is directly correlated with the trapstate density of perovskite film. Reducing the local traps would lead to higher CPD. We can therefore reasonably conclude that the fumigated perovskite film has a lower trap-state density compared with the reference one. The $\mathrm{CPD}$ increment in response to the light illumination suggests that the fumigated film has a mitigated potential loss for about $102 \mathrm{mV}$. The FWHMs of CPD peaks under illumination indicate that the fumigated film has a more uniform surface potential than that of the reference one, probably due to the decreased potential barrier along oriented lattice terminations [25]. Such statement can be further evidenced by the linear analysis of CPD fluctuation relative to the height profile (Fig. S14). As an extention, we studied the CPD distribution of perovskite films fumigated by other solvents under light soaking. As shown in Fig. S15, all fumigated films exhibit higher CPD than that of the reference one, while only the textured EA film has the optimal parameters (highest CPD and narrowest FWHM). Based on the above analytic results, we
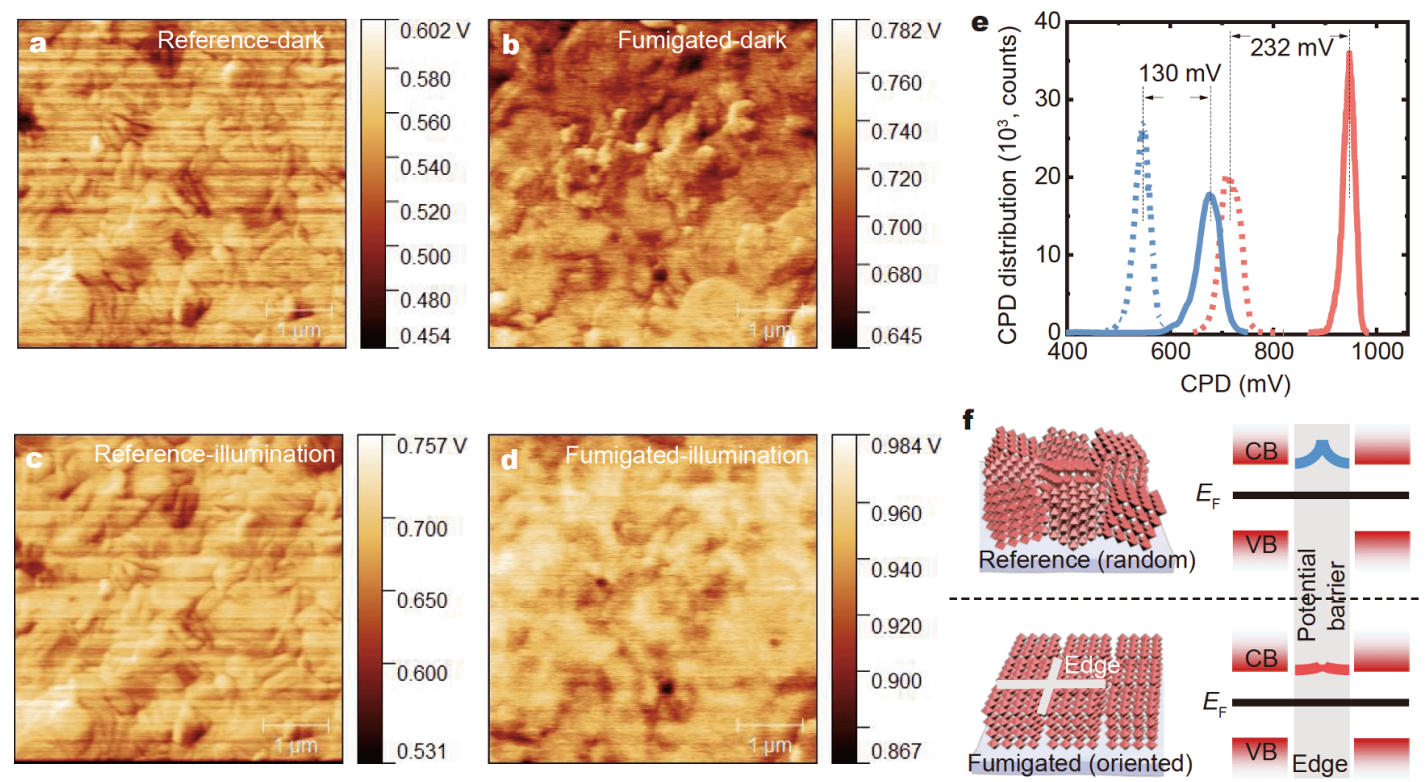

Figure 3 KPFM images of the reference and fumigated perovskite films ( $a, b)$ under dark and (c, d) under the same-intensity light illumination. (e) CPD distributions of the reference (blue) and fumigated (red) perovskite films (under dark: dashed line; under illumination: solid line). (f) Conceptual band models of random and oriented perovskite lattices. 
are able to conjecture a band model relative to the mesoscopic structure of perovskites under the operational condition (Fig. 3f). The oriented perovskite lattices upon fumigation treatment would render a decreased charge transport barrier throughout the film.

Steady-state PL spectroscopy was used to assess the charge recombination behavior of the as-prepared perovskite films. As shown in Fig. 4a, the PL intensity of the fumigated film at $\sim 653 \mathrm{~nm}$ is about 1.5 times stronger than that of the reference one, suggesting a suppressed non-radiative recombination for the fumigated film [28]. The bandgap of perovskite films determined by PL spectra is consistent with the one determined by ultraviolet-visible (UV-vis) spectra (Fig. S16), with a value of $\sim 1.9 \mathrm{eV}$. The time-resolved PL (TRPL) spectra in Fig. 4b show that the fumigated film exhibits prolonged carrier lifetime ( $80.2 \mathrm{~ns})$ than that of the reference one ( $46.9 \mathrm{~ns})$, which are obtained by biexponentially fitting the data and composed of both fast and slow components, again verifying the suppressed charge carrier recombination upon the fumigated perovskite film $[29,30]$.

Using vertical device configuration (glass/FTO/perovskite/Au), we further measured the space-charge current limited (SCLC) curves of perovskite films to study their electrical properties and quantitatively determine the trap-state densities $\left(N_{\mathrm{T}}\right)$. As shown in Fig. $4 \mathrm{c}$, the fumigated film exhibits a higher current density than the reference one over the entire bias region $(0-10 \mathrm{~V})$, indicating its improved conductivity which is probably due to the oriented structure [14]. We further measured the current-voltage $(J-V)$ curves of the devices under the bias region of -2 to $2 \mathrm{~V}$ to quantitatively evaluate the carrier mobility of perovskite films using Mott-Gurney law (Fig. S17) [31]. The as-obtained carrier mobility of the fumigated film $\left(9.7 \mathrm{~cm}^{2} \mathrm{~V}^{-1} \mathrm{~s}^{-1}\right)$ has increased about two fold compared with the reference one $\left(4.6 \mathrm{~cm}^{2} \mathrm{~V}^{-1} \mathrm{~s}^{-1}\right)$. In addition, the fumigated film has a trap-filled limit voltage $\left(V_{\mathrm{TFL}}\right)$ of $0.81 \mathrm{~V}$, lower than the $V_{\mathrm{TFL}}$ of $1.12 \mathrm{~V}$ for the reference one, suggesting its reduced $N_{\mathrm{T}}$ [32]. The $N_{\mathrm{T}}$ of perovskite films can be calculated as following [33]: $V_{\mathrm{TFL}}=q N_{\mathrm{T}} d^{2} / 2 \varepsilon \varepsilon_{0}$, where $q$ is the elemental charge, $d$ is the thickness of the perovskite film, $\varepsilon$ is the dielectric constant of perovskite, $\varepsilon_{0}$ is the vacuum permittivity. The $N_{\mathrm{T}}$ of the perovskite film decreases from $\sim 4.62 \times 10^{16}$ to $\sim 3.34 \times 10^{16} \mathrm{~cm}^{-3}$ after fumigation. Such results are in agreement with the CPD maps and are coincident with $V_{\mathrm{TFL}}$ measured from the dark current curves of electron-only device configuration (Fig. S18).

Above-mentioned characterization results have revealed the structural and photophysical properties of the perovskite films. We then measured the $J$ - $V$ curves of the
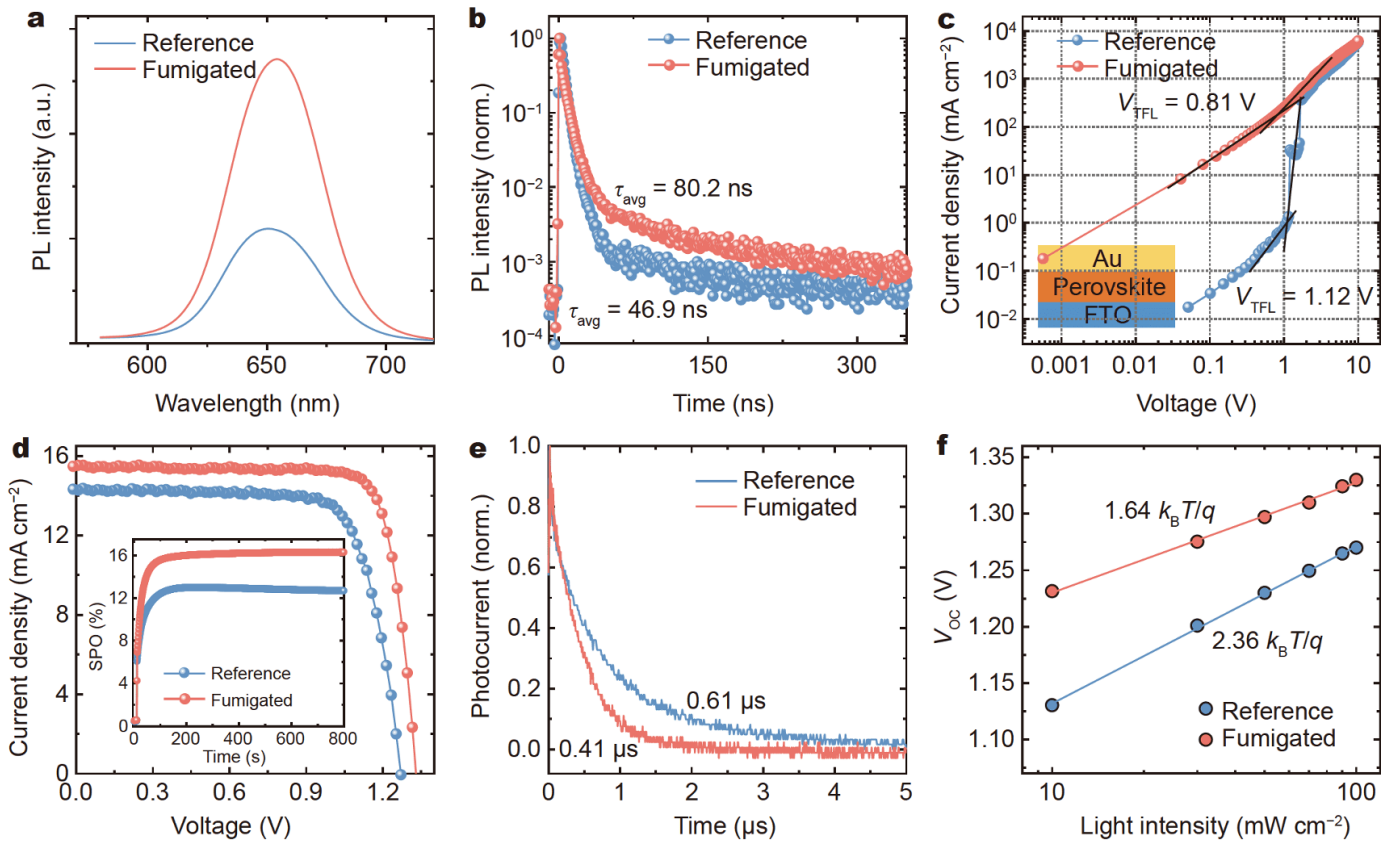

Figure 4 (a) PL and (b) TRPL spectra of the reference and fumigated perovskite films deposited on glass substrates. (c) SCLC curves of the reference and fumigated perovskite films. (d) $J$ - $V$ curves of the champion reference and fumigated PSCs. Inset is the MPP tracking of devices. (e) TPC curves of the reference and fumigated PSCs under $10 \mathrm{~mW} \mathrm{~cm}{ }^{-2}$ light-emitting diode (LED) illumination. (f) Dependence of $V_{\mathrm{OC}}$ on incident light intensity for the reference and fumigated PSCs. 
solar cells based on a planar n-i-p configuration (glass/ $\mathrm{FTO} / \mathrm{TiO}_{2} / \mathrm{CsPbI}_{1.8} \mathrm{Br}_{1.2} / \mathrm{P} 3 \mathrm{HT} / \mathrm{Au}$ ). The thickness of the perovskite film is estimated to be $\sim 300 \mathrm{~nm}$ (Fig. S19). All $J-V$ curves were recorded under simulated AM $1.5 \mathrm{G}$ irradiation with a scan rate of $0.1 \mathrm{~V} \mathrm{~s}^{-1}$. As shown in Fig. $4 \mathrm{~d}$ and Table S2, the champion fumigated device exhibited a short-circuit current density $\left(J_{\mathrm{SC}}\right)$ of $15.5 \mathrm{~mA} \mathrm{~cm}^{-2}$, an open-circuit voltage $\left(V_{\mathrm{OC}}\right)$ of $1.33 \mathrm{~V}$ and fill factor $(\mathrm{FF})$ of 0.806 , delivering a PCE of $16.6 \%$, and the $J_{\mathrm{SC}}, V_{\mathrm{OC}}$, FF and PCE of the reference one are $14.3 \mathrm{~mA} \mathrm{~cm}^{-2}, 1.27 \mathrm{~V}, 0.748$ and $13.6 \%$, respectively. Besides, the hysteresis of the fumigated device is reduced at forward scan direction (Fig. S20). The steady-state photocurrent outputs of the PSCs were tracked at a maximum power point (MPP). The photocurrents of the reference and fumigated devices were measured to be $\sim 12.7$ and $\sim 14.5 \mathrm{~mA} \mathrm{~cm}^{-2}$ at $800 \mathrm{~s}$ under ambient condtion, corresponding to the stabilized power outputs (SPOs) of $12.7 \%$ and $16.3 \%$ under the applied bias voltages of 1.00 and $1.12 \mathrm{~V}$, respectively. The EQE spectra are provided in Fig. S21, of which the integrated $J_{\mathrm{SC}}$ for the reference and fumigated PSCs are 13.5 and $14.7 \mathrm{~mA} \mathrm{~cm}^{-2}$, respectively, in agreement with the $J_{\mathrm{SC}}$ measured from standard $J-V$ curves. We found that fumigation treatment would optimize all photovoltaic parameters of PSCs (Fig. S22) and lead to an average PCE of $15.9 \%$, contrasted by the $12.3 \%$ of the reference ones. Such PCE enhancement can be probably ascribed to the largely improved crystal quality of the perovskite absorbers. The photovoltaic performances of the perovskite absorbers fumigated by MT, ET, IPA and TL are not as good as the one fumigated by EA (Fig. S23 and Table S2), in agreement with the CPD results of the perovskite films.

The TPC measurement was conducted to evaluate the charge collection efficiency of PSCs (Fig. 4e). The fumigated PSC exhibited a TPC lifetime of $0.41 \mu$ s, contrasted by the $0.61 \mu$ s of the reference one, indicating a more efficient charge collection of fumigated PSC [1]. However, the TPV lifetime of fumigated PSC is prolonged (Fig. S24), proving its reduced charge carrier recombination [1]. The interfacial charge transfer and recombination behaviors of PSCs were studied by impedance spectroscopy. As shown in Fig. S25, the radius of fitted semicircle of the fumigated device is larger than the reference one under the applied bias voltage of $1 \mathrm{~V}$, suggesting its enhanced resistance $\left(R_{\text {rec }}\right)$ for interfacial charge recombination. The identical results were observed within the bias voltage region from 0 to $1 \mathrm{~V}$. The dependence of $V_{\mathrm{OC}}$ on light intensity gives the ideality factor $\left(n_{0}\right)$ of PSCs, which was obtained by linearly fitting the data and can be further used to evaluate the re- combination of photogenerated carriers within the devices $[34,35]$. The reference device has a $n_{0}$-value of 2.36 , apparently higher than the $n_{0}$-value of 1.64 for the fumigated one and is beyond the boundary value of 2 (Fig. 4f), indicating that the trap-assisted recombination has dominated the reference device due to the nonideal perovskite film quality [35].

\section{CONCLUSIONS}

We have reported a crystallization regulation strategy on inorganic perovskite films by employing a fumigation treatment on colloidal precursors. The enhanced crystal quality of the resultant films is in coincidence with the previous reports $[2,36]$, i.e., inorganic perovskites may demand a slower crystallization to produce high-quality films for photovoltaics. The EA fumigation facilitates the formation of oriented perovskite lattices during liquid-tosolid transformation, which cannot be readily achieved upon other commonly used volatile solvents. Our results indicate that the atmosphere might play an important role on controlling the crystallographic features of solution-processed perovskite films. On the other hand, with the assistance of advanced in-situ monitoring technologies, the underlying impact of processing atmosphere on perovskite absorbers is worth being further explored (e.g., ambient air in the presence of water and oxygen, or other solvent vapors). A tailored processing atmosphere for perovskite absorbers might largely contribute to the development of high-performance PSCs targeting future commercialization.

Received 11 January 2021; accepted 22 February 2021; published online 15 April 2021

1 Wang Y, Dar MI, Ono LK, et al. Thermodynamically stabilized $\beta$ $\mathrm{CsPbI}_{3}$-based perovskite solar cells with efficiencies $>18 \%$. Science, 2019, 365: 591-595

2 Chen W, Chen H, Xu G, et al. Precise control of crystal growth for highly efficient $\mathrm{CsPbI}_{2} \mathrm{Br}$ perovskite solar cells. Joule, 2019, 3: 191204

3 Mali SS, Patil JV, Shinde PS, et al. Fully air-processed dynamic hotair-assisted $\mathrm{M}: \mathrm{CsPbI}_{2} \mathrm{Br}\left(\mathrm{M}: \mathrm{Eu}^{2+}, \mathrm{In}^{3+}\right)$ for stable inorganic perovskite solar cells. Matter, 2021, 4: 635-653

4 Yoon SM, Min H, Kim JB, et al. Surface engineering of ambientair-processed cesium lead triiodide layers for efficient solar cells. Joule, 2021, 5: 183-196

5 Liu C, Li W, Zhang C, et al. All-inorganic $\mathrm{CsPbI}_{2} \mathrm{Br}$ perovskite solar cells with high efficiency exceeding $13 \%$. J Am Chem Soc, 2018, 140: 3825-3828

6 Zhang L, Li B, Yuan J, et al. High-voltage-efficiency inorganic perovskite solar cells in a wide solution-processing window. J Phys Chem Lett, 2018, 9: 3646-3653

7 Mali SS, Patil JV, Hong CK. Hot-air-assisted fully air-processed barium incorporated $\mathrm{CsPbI}_{2} \mathrm{Br}$ perovskite thin films for highly 
efficient and stable all-inorganic perovskite solar cells. Nano Lett, 2019, 19: 6213-6220

8 Tian J, Xue Q, Tang X, et al. Dual interfacial design for efficient $\mathrm{CsPbI}_{2} \mathrm{Br}$ perovskite solar cells with improved photostability. Adv Mater, 2019, 31: 1901152

9 Lau CFJ, Deng X, Zheng J, et al. Enhanced performance via partial lead replacement with calcium for a $\mathrm{CsPbI}_{3}$ perovskite solar cell exceeding $13 \%$ power conversion efficiency. J Mater Chem A, 2018, 6: 5580-5586

10 Duan J, Zhao Y, Yang X, et al. Lanthanide ions doped $\mathrm{CsPbBr}_{3}$ halides for HTM-free 10.14\%-efficiency inorganic perovskite solar cell with an ultrahigh open-circuit voltage of 1.594 V. Adv Energy Mater, 2018, 8: 1802346

11 Liu C, Li W, Li H, et al. Structurally reconstructed $\mathrm{CsPbI}_{2} \mathrm{Br}$ perovskite for highly stable and square-centimeter all-inorganic perovskite solar cells. Adv Energy Mater, 2019, 9: 1803572

12 Sun $\mathrm{H}$, Zhang J, Gan $\mathrm{X}$, et al. Pb-reduced $\mathrm{CsPb}_{0.9} \mathrm{Zn}_{0.1} \mathrm{I}_{2} \mathrm{Br}$ thin films for efficient perovskite solar cells. Adv Energy Mater, 2019, 9: 1900896

13 Wang KL, Wang R, Wang ZK, et al. Tailored phase transformation of $\mathrm{CsPbI}_{2} \mathrm{Br}$ films by copper(II) bromide for high-performance allinorganic perovskite solar cells. Nano Lett, 2019, 19: 5176-5184

14 Kim DH, Park J, Li Z, et al. 300\% enhancement of carrier mobility in uniaxial-oriented perovskite films formed by topotactic-oriented attachment. Adv Mater, 2017, 29: 1606831

15 Qing J, Liu XK, Li M, et al. Aligned and graded type-II Ruddlesden-Popper perovskite films for efficient solar cells. Adv Energy Mater, 2018, 8: 1800185

16 Zhang X, Wu G, Fu W, et al. Orientation regulation of phenylethylammonium cation based 2D perovskite solar cell with efficiency higher than 11\%. Adv Energy Mater, 2018, 8: 1702498

17 Tsai H, Nie W, Blancon JC, et al. High-efficiency two-dimensional Ruddlesden-Popper perovskite solar cells. Nature, 2016, 536: 312316

18 Grob S, Bartynski AN, Opitz A, et al. Solvent vapor annealing on perylene-based organic solar cells. J Mater Chem A, 2015, 3: $15700-15709$

19 Wang JL, Xiao F, Yan J, et al. Difluorobenzothiadiazole-based small-molecule organic solar cells with $8.7 \%$ efficiency by tuning of $\pi$-conjugated spacers and solvent vapor annealing. Adv Funct Mater, 2016, 26: 1803-1812

20 Zhu X, Xu Z, Zuo S, et al. Vapor-fumigation for record efficiency two-dimensional perovskite solar cells with superior stability. Energy Environ Sci, 2018, 11: 3349-3357

21 Zhao X, Liu T, Kaplan AB, et al. Accessing highly oriented twodimensional perovskite films via solvent-vapor annealing for efficient and stable solar cells. Nano Lett, 2020, 20: 8880-8889

22 Zhou Z, Qiao HW, Hou Y, et al. Epitaxial halide perovskite-based materials for photoelectric energy conversion. Energy Environ Sci, 2021, 14: 127-157

23 Lin C-, Li S-, Chang J-, et al. Unveiling the nanoparticle-seeded catalytic nucleation kinetics of perovskite solar cells by time-resolved GIXS. Adv Funct Mater, 2019, 29: 1902582

24 Jiang CS, Yang M, Zhou Y, et al. Carrier separation and transport in perovskite solar cells studied by nanometre-scale profiling of electrical potential. Nat Commun, 2015, 6: 8397

25 Yun JS, Ho-Baillie A, Huang S, et al. Benefit of grain boundaries in organic-inorganic halide planar perovskite solar cells. J Phys Chem Lett, 2015, 6: 875-880

26 Lin YY, Chu TH, Li SS, et al. Interfacial nanostructuring on the performance of polymer/ $/ \mathrm{TiO}_{2}$ nanorod bulk heterojunction solar cells. J Am Chem Soc, 2009, 131: 3644-3649

27 Doherty TAS, Winchester AJ, Macpherson S, et al. Performancelimiting nanoscale trap clusters at grain junctions in halide perovskites. Nature, 2020, 580: 360-366

28 Qiao HW, Yang S, Wang Y, et al. A gradient heterostructure based on tolerance factor in high-performance perovskite solar cells with 0.84 fill factor. Adv Mater, 2019, 31: 1804217

29 Stranks SD, Eperon GE, Grancini G, et al. Electron-hole diffusion lengths exceeding 1 micrometer in an organometal trihalide perovskite absorber. Science, 2013, 342: 341-344

30 Chen SC, Zhang S, Zheng Q. A facile surface passivation method for efficient inorganic $\mathrm{CsPbI}_{2} \mathrm{Br}$ perovskite solar cells with efficiencies over 15\%. Sci China Mater, 2020, 63: 719-727

31 Yao K, Li F, He Q, et al. A copper-doped nickel oxide bilayer for enhancing efficiency and stability of hysteresis-free inverted mesoporous perovskite solar cells. Nano Energy, 2017, 40: 155-162

32 Duijnstee EA, Ball JM, Le Corre VM, et al. Toward understanding space-charge limited current measurements on metal halide perovskites. ACS Energy Lett, 2020, 5: 376-384

33 Rose A. Space-charge-limited currents in solids. Phys Rev, 1955, 97: $1538-1544$

34 Xie F, Chen CC, Wu Y, et al. Vertical recrystallization for highly efficient and stable formamidinium-based inverted-structure perovskite solar cells. Energy Environ Sci, 2017, 10: 1942-1949

35 Wetzelaer GAH, Kuik M, Nicolai HT, et al. Trap-assisted and Langevin-type recombination in organic light-emitting diodes. Phys Rev B, 2011, 83: 165204

36 Wang $\mathrm{P}$, Zhang $\mathrm{X}$, Zhou $\mathrm{Y}$, et al. Solvent-controlled growth of inorganic perovskite films in dry environment for efficient and stable solar cells. Nat Commun, 2018, 9: 2225

Acknowledgements This work was financially supported by the National Ten Thousand Talent Program for Young Top-notch Talent, the National Natural Science Fund for Excellent Young Scholars (52022030), the National Natural Science Fund for Distinguished Young Scholars (51725201), the National Natural Science Foundation of China (51972111 and 51902185), the International (Regional) Cooperation and Exchange Projects of the National Natural Science Foundation of China (51920105003), the Innovation Program of Shanghai Municipal Education Commission (E00014), the Fundamental Research Funds for the Central Universities (JKD012016025 and JKD012016022), and Shanghai Engineering Research Center of Hierarchical Nanomaterials (18DZ2252400). The authors thank the Frontiers Science Center for Materiobiology and Dynamic Chemistry. The authors also thank beamline BL14B1 of Shanghai Synchrotron Radiation Facility for providing the beamtime.

Author contributions Zhou $\mathrm{Z}$ designed and performed the experiments; Qiao HW, Xie J, Shi Y, Lin Z, Ge B and Chen M helped revise the paper; Li X helped perform the GIWAXS characterization; Lin C and Jin YZ helped perform the AFM/KPFM measurements; Yang S, Hou Y and Yang HG provided all supports needed in this work. All authors contributed to the general discussion.

Conflict of interest The authors declare no conflict of interest

Supplementary information Supporting data are available in the online version of the paper. 


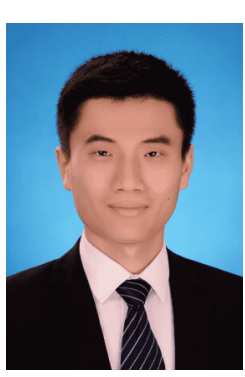

Ziren Zhou received his BS from Xiangtan University in 2017. He is currently a $\mathrm{PhD}$ candidate at the East China University of Science and Technology (ECUST) under the supervision of Prof. Hua Gui Yang. His research interest focuses on the design and synthesis of functional materials for perovskite-based photovoltaics.

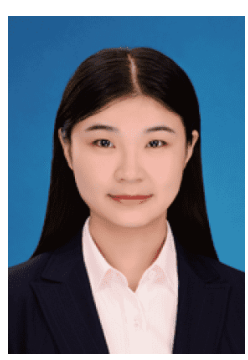

Hong Wei Qiao received her $\mathrm{PhD}$ from ECUST in 2020. Her research interest focuses on the design, synthesis and characterization of new semiconductor materials' thin films, and looking for their applications in solar cells.

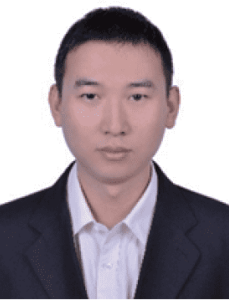

Shuang Yang received his BS from Tsingtao University of Science and Technology in 2011 and completed his PhD in 2016 from ECUST. He then conducted postdoctoral research at $\mathrm{Na}$ nyang Technological University and University of Nebraska-Lincoln. Currently, he is a professor at ECUST. His research focuses on the chemistry and physics of semiconducting materials and opto-electrical devices.

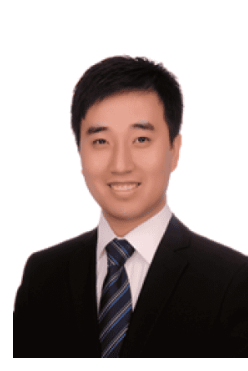

Yu Hou completed his BS and PhD from ECUST in 2010 and 2015, respectively. From 2015 to 2017, he worked as a postdoctoral research fellow at ECUST and then joined Griffith University as a visiting scholar. After finishing his visiting scholar training, he came back to ECUST at the end of 2017 and now is a professor at ECUST. His research focuses on novel functional materials of thin film-based solar devices and aims at developing new semiconductor absorbers and cell structures towards next-generation photovoltaic technology.

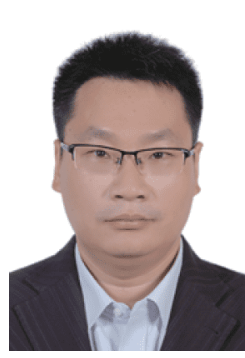

Hua Gui Yang completed his $\mathrm{PhD}$ from the National University of Singapore in 2005 . He joined the University of Queensland in 2007 as a postdoctoral research fellow. After finishing his postdoctoral training, he came back to China and took up a professor position at ECUST at the end of 2008. His research interests are focused on the rational design and fabrication of functional materials for solar-energy conversion.

\section{溶胶态熏蒸处理制备取向规整的无机钻钛矿吸光 层薄膜}

周资人 ${ }^{1 \dagger}$, 乔红伟 ${ }^{1 \dagger}$, 李晓龙 ${ }^{2}$, 林晨 $^{3}$, 谢瑾 ${ }^{1}$, 施以恒 ${ }^{1}$, 林泽青 $^{1}$, 葛兵 ${ }^{1}$, 陈锰畑 ${ }^{1}$, 金一正 ${ }^{3}$, 杨双 ${ }^{1 *}$, 侯宇 ${ }^{1^{*}}$, 杨化桂 ${ }^{1}$

摘要 全无机钙钛矿卤化物拥有优良的光、热稳定性, 被认为是颇 具潜力的新一代光伏材料. $\mathrm{CsPbI}_{x} \mathrm{Br}_{3-x}$ 作为一种卤素混合的钻钛矿 成分在结构容忍性和光吸收带边两者之间取得了平衡, 然而其通 过溶液加工获得的吸光层薄膜结晶质量通常情况下不够理想, 使 得无机钙钛矿太阳能电池的光电转换效率受到限制. 本论文提出 了一种基于无机钻钛矿薄膜的结晶调控手段, 即利用双氧供体的 乙酸乙酯蒸汽熏蒸处理溶胶态无机钙钛矿前驱体薄膜. 通过广角 掠入射X射线散射谱原位表征，我们发现该熏蒸处理手段能够抑制 溶胶态钻钛矿的熟化, 从而降低钻钛矿的形核速度. 高温退火后的 钙钛矿薄膜获得了较高的结晶度和规整的晶体取向, 并表现出良 好的光电特性, 基于此制备的宽带隙 $(1.9 \mathrm{eV})$ 无机钙钛矿太阳能电 池取得了 $16.6 \%$ 的光电转换效率. 